\title{
A Study on Intelligent Traffic System related with Smart City
}

\author{
Byung-Tae Chun ${ }^{1}$ \\ ${ }^{1}$ Dept. of Web Information Engineering, Hankyong National University, 327, \\ Chungang-no, Anseong-si, Kyonggi-do, Korea \\ chunbt@hknu.ac.kr
}

\begin{abstract}
Digital convergence is spoken of basically as convergence of various information and communication technologies. The significance and waves for digital convergence, however, do not remain merely in the boundary of chemical combinations or increase of economic efficiency. The core technology of the large technological direction is smart, mobile. In this paper, we described intelligent traffic system which was leading case in digital convergence and related with our life. Intelligent Traffic System (ITS) integrates cutting-edge technology into existing traffic system elements. We described actual cases, analysis and applicability of current ITS.
\end{abstract}

Keywords: ICT, Smart, Intelligence, Actual Cases

\section{Introduction}

In close connection with the term, 'digital,' 'convergence' is a word of the times in this information society that indicates new products and services or trends that emerge out of integration or reflection of unit technologies of the general information and communication sector [1]. Digital convergence is spoken of basically as convergence of various information and communication technologies, media, commodities, and services, convergence of hardware units or software units, convergence between hardware and software, convergence between humans and machines, and convergence between virtual and physical spaces [2]. The significance and waves, however, do not remain merely in the boundary of chemical combinations or increase of economic efficiency. As multiple technologies and media or cultural elements go through a sort of advancement and convergence, the boundary among them becomes vague up to the point that even classifying them is meaningless. As a result, a new social order and cultural phenomena emerge, which changes the general aspects of our daily lives.

There have already been clear signs of the ripple effects of convergence technology and a new convergence society over economics, society, culture, and politics. In integration of cutting-edge science technology into architecture and civil engineering, Intelligent Building and Smart Highway are only two examples of convergence that have been embodied. Digital convergence means to create new products or services through integration of unit technologies based on information and communication technology. Such digital convergence meant simple functional integration of technologies and products in the past, but recently, it is advancing toward a higher level of industrial development and creation of new industry sectors in integration of IT into existing industry as well as organic combination among products and services. 


\section{Intelligent Traffic System}

Smart city means a city where public functions available in common cities are networked by means of cutting-edge information / communication technology. Functions necessary for the realization of ICT-based smart city include concepts of sensor-based ubiquitous environment and intelligence [3, 7, 8]. Smart city is, in other terms, a futureoriented advanced city that is pursued around the world as the fruit of ICT-based convergence industry. To realize such smart city, a large-scale project widely applicable from basic industries such as environment, transportation, utility, and construction to specific home appliances need to be developed. The element of smart city, as shown in Table 1, consists of the six: smart government, smart building, smart mobility $[5,6,9]$, smart energy, environment and smart service.

Table 1. Functions of Six Elements

\begin{tabular}{|c|c|}
\hline Elements & \multicolumn{1}{|c|}{ function } \\
\hline Smart Government & - Support necessary services to citizen \\
& - Management of various infrastructure \\
\hline Smart Energy & - Means smart grid \\
& - Basic energy infrastructure \\
\hline Smart Environment & - Environmentally-friendly factors \\
& - Green IT \\
\hline Smart Building & - Provide clean and economic environment \\
\hline Smart Mobility & - Traffic services with more intelligences \\
& - Optimize the traffic flow \\
\hline Smart Services & - Service type provided to citizen \\
& - Environment, learning, culture, etc \\
\hline
\end{tabular}

Smart Mobility is a concept of comprehensive and smarter future traffic service in combination with Smart Technology. A smart mobility society is realized by means of the current intelligent traffic systems.

Intelligent Traffic System integrates cutting-edge technology into existing traffic system elements such as road, vehicle, and signaling system in order to improve the traffic facility, efficiency and safety [5, 6, 9]. Just as a human brain controls and adjusts the physical body, the intelligent traffic system controls the traffic facilities flexibly and automatically based on traffic information acquired from various channels for user convenience to the full.

This type of ITS controls signals in reflection of the volume of traffic, regulates over-speed vehicles, collects fees on express ways automatically, provides traffic information for smooth traffic flows, provides information of times of bus arrivals and routes, and additionally provides information of other transportation means such as subway, train, and flight as well as vehicles so that users can be informed of the current traffic situation anytime and anyplace.

Currently, most drivers have no difficulty in finding the destination by using a navigation system even if they do not know the region much. In addition to finding the optimal route, this system recognizes traffic congestions in advance and finds better ways. While waiting at a bus stop, the user can find traffic information on when the next bus will arrive, how long it will take from there to the destination, etc. Intelligent traffic system makes such information available. 


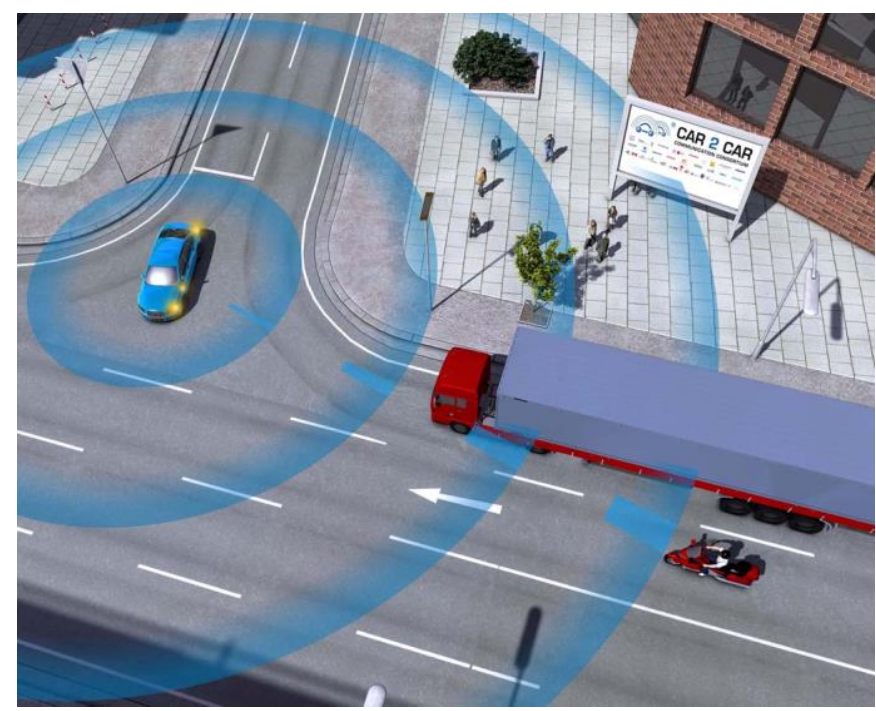

Figure 1. Example of Intelligent Traffic System

\section{Oversea Cases of ITS}

It is known that the intelligent traffic system in Austria sector is developing fast owing to the government's active supporting and a lot of efforts for technological development. ITS, which is also called automotive telematics, provides vehicles and drivers with necessary information and service by means of communication devices in the vehicle based on a location information system that utilizes wireless voice signals, data communication, and artificial satellites. In its initial state of development, the single term, telematics,' was used, but since 2009 when telematics was converged with ITS, it was called 'tele-convergence' and since 2010, 'smart traffic' also has been used. Based on a wealth researches, related sectors are progressing rapidly.

When it comes to ITS, the technical power Austria is widely recognized, and the dynamic force is the governmental support and strong will. One example is 'National ITS Action Plan 2011,' which initiated a test operation of ITS in expressways and outer rings of Wien, the capital city.

A vehicle on the move is both a supplier and user of useful traffic information as it is equipped with a navigation system, cameras, and sensors while control centers collect, apply, and provide such information.

The Austrian government has carried forward its project to develop and foster the ITS sector since the early 2000s. In 2002, the strategic program for intelligent traffic system and service (IV2S) was planned, and in 2004, the government announced its telematic strategic plan. In 2005, as part of the related technology development program, Austria Tech GmbH was founded under BMVIT, Austria. In 2007, existing IVS programs were modified in harmony with EU-related programs. In 2008, Austria BMVIT took the lead of ITS Vienna Region, a pilot project initiated in association with Wien, Burgenland, and Niederösterreich. The peak of such governmental efforts was 'National ITS Action Plan 2011' released in November 2011. This combines all elements of ITS Austrian policies. The primary three objectives of this plan are as follows: First, all traffic-related information throughout Austria is integrated and standardized in its traffic graph; second, EGovernment system is utilized to develop and use an effective solution to making use of and control traffic information; lastly, a standardized platform or an integrated traffic information system is established to provide traffic information real time. 
Major organizations related to Austria ITS include Austria Traffic Telematics Cluster, which was founded in 2003 for the development of Austria telematics sector. Research centers and companies that belong to this organization have formed a systematic, cooperative relation with the aim to develop new technology applicable to the traffic management telematics system. Besides, Austria Tech, Kapsch TrafficCom, and Frequentis have been recognized of their technical power in related sectors. Austria Tech, a company under Austria BMVIT, has been in charge of ITS core technology development since its foundation in 2005. Kapsch TrafficCom is one of the world leading companies in the area of road traffic telematics. Kapsch TrafficCom under Kapzsch Group provides various solutions such as real-time traffic information in association with Kapsch Telematic Services. Such systems contribute to reducing costs related to road maintenance as well as providing more stable and better services. Frequentis is a worldly renowned company in the area of aerial traffic management and control system. In various areas such as private and military aerial traffic control, public safety (police, ambulance, fire, etc.), railroad, and marine system, it has supplied solutions in more than 80 countries around the world. This organization is well known for the technical power in the areas of voice-based system for aerial traffic control, providing its solution to Incheon International Airport, Korea.

The Indian government is expanding its investment into city public traffic infrastructures in cooperation with the World Bank, etc. Today, the public roadway system of India includes $3,320,000 \mathrm{~km}$ over the country, including federal state road, expressway, local and rural roads, etc., which is the second largest in the world. The total length of railroad in that country is $64,099 \mathrm{~km}$, which is the fourth in the world and the second in Asia. 94\% of the roadways in India, however, are local and rural roads, and $50 \%$ of the federal state roads managed by the government are two-lane roads $30 \%$ one-lane roads, and $17 \%$ only $4 / 6 / 8$-lane roads. As for railroad as well, the traffic network infrastructure is in a poor condition with frequent accidents due to defective and old facilities such as delay, derailment, collision, fire, etc.

Since 2007, the Indian government is conducting a project to expand public transportation facilities such as bus, train, and electric car to the 24 major cities although many cities with a population of 2 million have simply no infrastructure of public transportation. Above all, the traffic infrastructure cannot keep up with the rapidly increasing number of vehicles, and thus the introduction of intelligent traffic system seems to be the very basis for effective and efficient traffic system. The increase rates of vehicles in India are presented in Figure 2 below:

Against this background and in recognition of the need for policies to handle serious traffic congestions and lack of public transportation facilities in response to the rapid urbanization, the Indian government introduced a new policy called JNNURM (Jawaharlal Nehru National Urban Renewal Mission). JNNURM aims to provide basic transportation services and develop major infrastructures for poor citizens in 61 major cities by investing 20 billion dollars.

Specifically, the policy includes basic traffic system for poor citizens, LED signboard, automatic ticket barrier, voice direction, smart card, CCTV, automatic GPS, communication service, etc., which are all based on the utilization of ITS. In addition, NUTP (National Urban Transport Policy) announced by the Indian government in 2006, aims at establishing effective and safe public transportation infrastructures. It includes replacement of old buses and introduction of ITS technology for convenient use of bus system.

In some of the major cities in India such as New Delhi and Mumbai have sought to introduce their own ITS system, but other cities that are incapable of doing that are supported by the government. The government has established SUTP (Sustainable Urban Transport Project over 5 of such cities and provided support for 
the introduction of ITS system. 0.17 billion dollars are planned to be invested for SUTP by the Indian federal government, the World Bank, and central government.

Mumbai, a major commercial city of India, is suffering from serious traffic congestions, frequent accidents, and air pollution due to poor road infrastructures rapidly increasing vehicles. With these problems hindering the growth of national economy, the central government has introduced ITS in various sectors. Major ITS projects of Mumbai are as follows:

The first is Traffic Management System. In utilization of the smart mobility solution developed by Telvent, an IT solution company, developed, Adaptive Traffic Control System (ATCS) sensors are installed over sections of traffic congestion to transmit information traffic conditions real time. Additionally, 600 cameras with geographical information system were installed all over the city to check the spots of traffic congestion in differentiated colors on the screen real time. It is reported that after the introduction of ATCS, traffic congestion has reduced as much as $25 \%$. In addition, Telvent is considering a smart grid project to supply electric power to roads and establish a distribution network.

The second is Intelligent Ticketing Solutions in Buses. In association with TRIMAX, an IT infra company, it has developed a bus information system and 'BEST" intelligent ticketing solution. This solution makes possible available online group ticketing and large-scale call bus service. Besides, bus route and account monitoring is more flexible and free ride is prevented.

The third is introduction of SMART Cards. Card terminals are installed for all types of transportation means such as bus, train, electric railway, taxi, etc., and a smart card system is introduced.

In an attempt to grasp and address the current poor traffic infrastructures, the Indian government is accelerating the progress of its project to establish ITS system in cooperation with global agencies such as the World Bank and UNDP as well as private companies such as Mott MacDonald and Telvent. As for drastically increasing needs for vehicles and public transportation means, road and railroad construction projects are viewed as highly profitable in India, and the Indian government announced its plan to establish transportation infrastructures and ITS over 200 cities nationwide.

The traffic infrastructures in Singapore are divided to road, MRT, and marine traffic. Most national territories of Singapore including such islands as Sentosa and Jurong are connected through the road network, and MRT (Mass Rappid Transit), the national subway system of Singapore, and LRT (Light Rail Transit) are connected through the NR Line and Circle-Line. Ferryboat services are also available for inter-island transportation.

According to LTA, the volume of traffic in Singapore will be increasing by 2020 $60 \%$ more than now. In consideration of the limited national territory of Singapore, an efficient public transportation system was essential in response to the increasing volume of traffic, and accordingly, a master plan for ITS system advancement was established in 2008.

LTA, an agency for inland transportation development in Singapore, is a statutory board under the Ministry of Transportation. It was established on September 1, 1995, in integration of four related organizations. Its mission is to satisfy various demands by providing a people-centered inland transportation system that is effective and cost-efficient, and its vision is to establish a people-centered transportation system accordingly. Cetrac ${ }^{\circledR}$ transportation management system, developed by Singapore Technologies Electronics Ltd and patented, is a primary system of Singapore ITS to which all ITS information is converged. This system In utilization of advanced image-processing technology and traffic engineering know-how, real time image signals and GPS traffic information are processed and analyzed. In addition, 
Singapore road management system was designed to optimize the number of vehicle users and assure smooth traffic flows.

Directions for Singapore ITS policies may be divided mainly into two: First, efforts are put into establishing intelligent information and communication infrastructures. With the aim to establish the next-generation national information and communication infrastructure that includes complementary wireless/cable networks, Singapore is planning to build up a ultra-high speed $1 \mathrm{Gbps}$ broadband in combination with a nationwide wireless network by 2015. Once an intelligent information and communication infrastructure that is constant and reliable is completed by 2015 , a wider and advanced road for the flow of digital resources will be opened, which will contribute to improving the general competitiveness in the area of information and communication. To promote $R \& D$ and innovation in emerging areas such as transportation, distribution, and media, and to induce strategic use of information and communication technology, the Singapore government is seeking cooperation among public and private agencies. Another aspect is to improve the charging mechanism of traffic congestion fees. Step 2 of ERP project is to improve the existing ERP system by deducting tolls from a driver's cash card inserted into IU (in-vehicle unit) at a toll gate. Basically, ERP 2.0 aims to realize a variable traffic congestion system in which the amount of traffic congestion fees is determined depending on how long a driver remains on the road rather than when he enters the road. The position of a driver is observed through IU with advanced GPS system. Ideally, real-time GPS tracking is supposed to be the basis with no longer need for ERP toll gates. As for GPS tacking, vehicles passing roads with heavy traffic to park in an office building and delivery vehicles moving on roads for hours are classified and more fees of traffic congestion are charged on vehicles staying on the roads longer, which contributes to reducing traffic congestion more effectively. Besides, its accuracy will be superior to that of the current fixed toll gate systems.

Primary concern of the inland transportation master plan of Singapore includes traffic congestion management system, location tracking system, and bus/subway management system. Thus, there may be ample chance for Korean small/medium SI companies to advance into the markets of monitoring devices such as camera, scanner, sensor, optical cable, accessories, etc.

Toyota City, Japan, November 26, 2014 - Starting next year, some of Toyota Motor Corporation's new models will be compatible with advanced vehicleinfrastructure cooperative systems that use a wireless frequency reserved for Intelligent Transport Systems. This compatibility will be offered as an option for the "Toyota Safety Sense P" active safety package that will be made available in 2015 on select new models sold in Japan.

The systems will use the dedicated ITS frequency of $760 \mathrm{MHz}$ for road-to-vehicle and vehicle-to-vehicle communication to gather information that cannot be obtained by onboard sensors. At intersections with poor visibility, information about oncoming vehicles and pedestrians detected by sensors above the road will be conveyed via road-to-vehicle communication, and information about approaching vehicles will be conveyed via vehicle-to-vehicle communication, with audio and visual alerts warning drivers when necessary.

Toyota believes that the ultimate goal of a society that values mobility is to eliminate traffic fatalities and injuries. Toyota's Integrated Safety Management Concept sets the direction for safety technology development and vehicle development, and covers all aspects of driving by integrating individual vehicle safety technologies and systems rather than viewing them as independently functioning units. This approach is reflected in $R \& D$ focusing on safety devices and systems. 
Toyota is firmly committed to a wide range of initiatives addressing traffic safety, ranging from the development of ever-safer vehicles and technologies to helping create a safe traffic environment and engaging in educational activities.

\section{Conclusions}

Intelligent traffic system combines existing system elements such as road, vehicle, and signaling system with cutting-edge technology such as electronics, controlling, and communication in order to enhance the efficiency of transportation facilities as well as safety. Based on traffic data acquired through various channels, a traffic system included artificial intelligence controls traffic condition automatically depending on the available transportation facility, which contributes to convenience of users.

'National ITS Action Plan 2011' in Austria reflects core elements of ITS policies of Austria. The technical power and growth potential of Austria ITS sector has been widely recognized around the world. Efforts need to be put forth into acquiring the advanced technologies among companies including those listed in this study particularly in such areas as GPS, camera, sensor, etc.

As urbanization progressed rapidly, the Indian government recognized the urgent need for policies addressing traffic congestion and insufficient public transportation means and introduced a new policy entitled JNNURM (Jawaharlal Nehru National Urban Renewal Mission). This policy aims to provide basic transportation services and develop major public transportation infrastructures by investing 20 billion dollars.

As for the direction of ITS policies that Singapore is currently working on, the two major aspects - establishment of intelligent information and communication infrastructures and improvement of fee collecting systems - are taken into account.

\section{References}

[1] NIA, "Convergence between IT based Industries and Strategy direction of National Information", (2010).

[2] J. Hangbae, "Future of Smart Technology", LG CNS, (2010).

[3] NIA. "Future City through Smart City" IT\&Future Strategy, (2010).

[4] S. H. Lee, D. W. Lee, “A Study on Digital Convergence and Smart City”, Journal of Digital Policy \& Management, vol. 11, no. 9, (2013), pp. 167-172.

[5] L. Figueiredo, I. Jesus, J. A. T. Machado, J. R. Ferreria and J. L.Carvalho,"Towards the development of intelligent transportation Systems", Proc. IEEE Intelligent Transportation Systems,(2001), pp. 12061211.

[6] F. Y. Wang, C. Herget and D. Zeng."Developing and Improving transportation systems: The Structure and operation", IEEE Trans. Intelligent Transportation Systems, vol. 6, no. 3, (2005), pp. 261-264.

[7] Pike Research,"IBM Raises the Stakes in the Smart City Technology market with its Intelligent Operations Center," (2011).

[8] Navigant Research, “Smart City”, (2013).

[9] R. Uzcategui, A. M. Guillermo, "WAVE, a tutorial," Communications Magazine, IEEE, (2009), pp. 126-133.

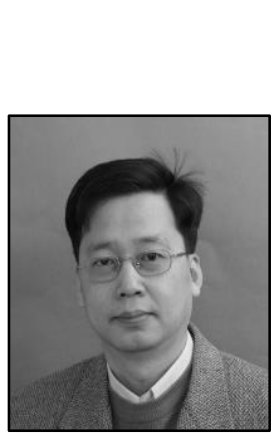

\section{Author}

Byung-Tae Chun, (PhD'11) He received the Ph.D. in computer engineering from Korea University, in 2011. From 1989 to 1996, he was a researcher at KIST(Korea Institute of Science and Technology). From 1996 to 2004, he was a senior researcher at Electronics and Telecommunication Research Institute). Since 2004, he has been a professor at Hankyong National University, Korea. He is a member IEEK and KIIT. 
International Journal of Smart Home

Vol. 9, No. 7 (2015) 